\title{
Operational and Performance Report on ANIPET, a Versatile PET Scanner for Laboratory Animals
}

\author{
K Q Nguyen*, C J Thompson*, S Kecani ${ }^{+}$, L Nikinnen ${ }^{+}$, \\ A Ben el Fassi, A Reader" N Zhang* and M Diksic* \\ Montreal Neurological Institute ${ }^{*}$ and Dept of Physics ${ }^{+}$, McGill University QC Canada, \\ Manchester Institute for Science and Technology", Manchester UK
}

\begin{abstract}
We present the properties and the performance of the ANIPET scanner which has been designed for imaging positronemitting tracers in small laboratory animals. It has two planar BGO-PS-PMT detectors with a useful FOV of 59x54 mm. These can be separated by $75-200 \mathrm{~mm}$ to optimize detection efficiency with different sized laboratory animals. ANIPET has the capacity to scan in 3D mode for brain images or 2D mode for whole body scanning. The spatial resolution at the centre of the field of view is $2.8 \mathrm{~mm}$ FWHM for 2D mode scans and $3.2 \mathrm{~mm}$ in the 3D mode. The coincidence timing resolution is $10 \mathrm{~ns}$ FWHM. The system efficiency varies from $1 \%$ to $0.1 \%$ as the detector separation increased from 75 to $200 \mathrm{~mm}$ and the energy discriminator window is increased from 150 to $450 \mathrm{keV}$.
\end{abstract}

\section{INTRODUCTION}

Animal models are very valuable in the study of basic human physiology and in developing treatments for diseases. Imaging techniques like positron emission tomography (PET) are becoming more widely available and permit in vivo studies that will complement the traditional techniques such as autoradiography [1]. Clinical research topics, such as the Serotonergic Theory of Affective Disorder and its pharmacological treatments, currently use invasive and laborious techniques such as autoradiography to measure tracer distribution in animal models [2]. Although ANIPET cannot attain the $100 \mu \mathrm{m}$ resolution of autoradiography, it will allow for more significant and complete in vivo studies of brain physiology and pharmaceutical distribution and kinetics [1].

The ANIPET scanner, by virtue of its small detector size, its optimized bismuth germanate (BGO) crystal pixelation and scintillation design, and its reconstruction software, can quantitatively measure tracer distribution in small volumes. Its detectors have a variable separation distance and can be positioned close to a subject without the effect of $\gamma$-ray scattering, which is minimal in a small animals. Small detector separation increases the sensitivity of the system and reduces the number of detectors required and subsequently the complexity and cost of the ANIPET is minimized [3].

There are several dedicated small animal PET scanners currently in use, all of which consist of many more detectors, typically configured as rings or co-planar blocks, and require large gantry support and complex electronics. As opposed to ANIPET, the TierPET [4] uses 4 YAP-block (Yittrium Aluminum Pervskit) detectors which rotate $90^{\circ}$ around a subject.
TierPET's maximum sensitivity is $1.75 \mathrm{kcps} / \mu \mathrm{Ci} / \mathrm{ml}$ (measured with a $5 \mathrm{~cm}$ diameter flood source) and its field of view (FOV) is 40x40 mm. ANIPET has only two detector blocks which are more sensitive than the TierPET and provide a larger FOV, but in many respects the systems are quite similar. The UCLA MicroPET [5] and the University of Sherbrooke Avalanche Photodiode-PET [6] use a ring detector configuration that remains stationary which permits faster dynamic scan framing. They have FOVs of $112 \times 18 \mathrm{~mm}$ and $118 \times 10.5 \mathrm{~mm}$, respectively. They are very sensitive over a small field of view (APD-PET: $2.0 \mathrm{kcps} / \mu \mathrm{Ci} / \mathrm{ml}$ for a $10.8 \mathrm{~cm}$ diameter flood source), but also more expensive.

\section{MATERIALS}

\section{A) System Description}

The ANIPET design, geometry, and components have been described previously [3][7]. Briefly, the system consists of two planar detectors operated in coincidence. These detectors are mounted on a horizontal metal shaft which is rotated by a stepping motor (Fig.1). The subject bed is mounted on a translation stage which is also driven by a stepping motor. The movement and position of these motors are controlled and monitored by the ANIPET acquisition software.

ANIPET has two scanning modes: 2D-axial and 3Drotary. The 2D-axial mode produces seven focal-plane images by backprojecting an inverse probability weighted value into the intersection of each plane with the line of response joining the coincident crystals. This technique has been used for breast cancer imaging in PEM-1 [8]. In this mode the scanner acquires data through a detector FOV of 59x54 mm but a whole body scan can be performed by moving the subject bed up tp $256 \mathrm{~mm}$ through this FOV. This produces a reconstructed image with a $256 \times 54 \mathrm{~mm}$ FOV.

In the 3D-rotary mode the detectors rotate around a cylindrical volume, which is determined by the detector offset from the axis of rotation and the detector separation (Fig. 1). When the detectors are not offset there is a 59 (axial) by $54 \mathrm{~mm}$ (radial) FOV, which is reconstructed and displayed off-line as 64 cross-sectional images of $64 \times 64$ pixels (scalable to $0.5,1.0$ or $2.0 \mathrm{~mm}$ square-pixels). The detectors can be offset from the axis of rotation by up to $25 \mathrm{~mm}$ to increase the radial FOV from 59 to $84 \mathrm{~mm}$. When the detectors are offset, the central region is sampled twice during a $360^{\circ}$ rotation. This operating mode is used in the CTI-RPT-2 [9]. The detectors can rotate as fast as $40^{\circ}$ per second, which allows for the minimal $180^{\circ}$ sampling to 

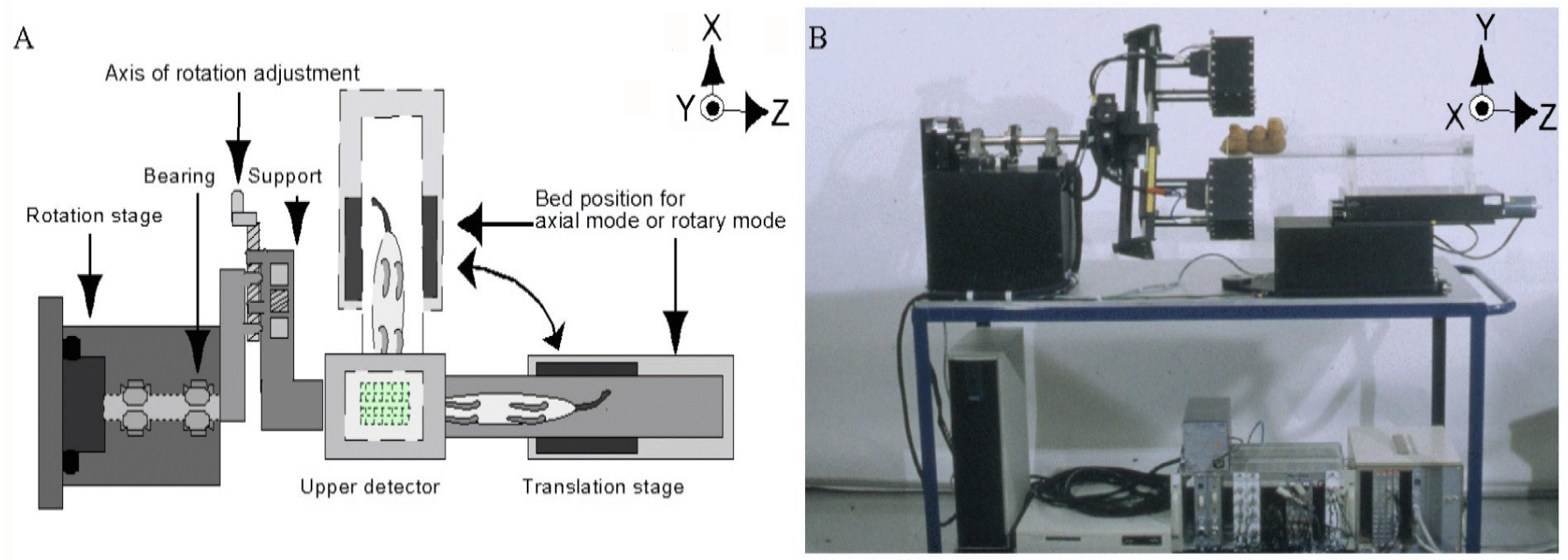

Figure 1: Schematics of the ANIPET Detectors and Translation Stage. A) Top view of the relative translation stage positions: the 3D-Rotary mode (solid); the 2D-axial mode (broken). B) Side view in the Rotary scanning mode where the detectors are rotating around the Y-axis. The entire system, including data acquisition electronics and computer, is contained on a mobile $0.8 \times 1.5 \mathrm{~m}$ cart.

be completed in the short frame times required during dynamic scanning protocols.

\section{B) ANIPET Hardware}

Each detector module consists of a $36 \times 36 \times 20 \mathrm{~mm}$ pixilated BGO crystal array coupled to a $72 \mathrm{~mm}$ square positionsensitive photomultiplier tube (PS-PMT Hamamatsu R3941-5). The crystals are cut from top and bottom surfaces into an offset, bi-layer array of $1.8 \times 1.8 \mathrm{~mm}$ pixels. The saw-cuts are filled with white epoxy filler to guide the scintillation light inside each element to the PS-PMT surface [7]. $\gamma$-rays are detected within the first layer of crystal elements, which are $6.5 \mathrm{~mm}$ in depth, or in the second layer of crystals (coupled to the PS-PMT), which are $11.5 \mathrm{~mm}$ in depth. These depths are based on Monte Carlo simulation results and ensure an equal probability of $\gamma$-ray interaction in each of the two layers, thereby providing 1-bit depth of interaction information, which is used to reduce the effect of radial-blurring.

\section{C) Data Acquisition}

There are four output signals from each detector $\left(\mathrm{X}^{-}, \mathrm{X}^{+}\right.$, $\left.\mathrm{Y}^{-}, \mathrm{Y}^{+}\right)$that are amplified and then subtracted to produce a total of six analog signals corresponding to the raw X-position, Yposition and energy signals of each incident event on a crystal face. Each detected $\gamma$-ray also evokes a timing signal from the last PMT dynode that is input to a constant fraction discriminator, which in turn generates a timing pulse. Coincident timing pulses are used to generate an ADC strobe signal from a coincidence module. The ADC strobe triggers the six channel 12-bit ADC to digitize the $\mathrm{X}, \mathrm{Y}$ and energy signals of each detector for all coincident events. These values are then saved into a list-file corresponding to the bed or detector position, and the time of the coincidence event.

There are also software calibration paradigms [7], which allow for the generation of look-up-tables (LUT) of element-toelement variations of efficiency, gain and spatial distortion.
These LUTs are used to perform corrections on element efficiency, energy discrimination and to convert PMTpositioning signals into spatial coordinates. The spatial distortion mapping is particularly important because it assigns the PS-PMT signals to individual crystal elements. The identification of the crystal elements is important for defining the size of the useful field of view and effects the absolute spatial resolution. This has recently been improved by modifying the cross anode readout resistor chain and the timing amplifier circuit in the PS-PMT [10].

\section{D) Scanning Protocols}

As with most PET scanners, ANIPET data is collected according to scanning protocols. Each scan protocol has animalspecific, system-specific, and scan-specific sections which provide a precise description of how to perform the scan. The animal-specific section identifies the animal species, researcher, tracer type, quantity, time and route of administration. The system-specific section includes the rotation or axial scan limits and period, the energy discriminator settings and initial file size. The scan-specific section allows for up to six groups of up to 20 frames each. Each section of a protocol is saved as a header in the list file before each study starts. The frames in each group all have the same duration, and these durations must be a multiple of $1 / 2$ of the detector movement period. The protocols can be saved and retrieved to serve as prototypes for subsequent scan procedures. The animal-specific header becomes "fixed" once an acquisition commences. However, the system-specific and scanspecific headers can be edited prior to reconstruction. This allows re-processing of data with different energy windows, detector calibration factors, and/or data framing of dynamic studies.

\section{E) Data Formatting Prior to Reconstruction}

During the acquisition of a scan, the program tracks the position of the (rotary or axial) translation stage as a function of 
time and event number in one file, and saves a list of detected events ( six bytes per event) in another file. The list mode data can be re-framed in order to generate any sensible image set, as long as the each frame is a multiple of $1 / 2$ of the period of the detector movement period. Prior to reconstruction, each event is assigned to a pair of crystal elements, and a pair of (-ray energies using the LUTs. If the line of response (LOR) is within the field of view, the angle of the LOR is within the preselected range and the energies are within the preselected range, the LOR is then converted to parallel-projection in coordinate space. The parallel-projection files are used to generate sinograms which are used by the reconstruction algorithm.

In order to handle multiple frames associated with a dynamic study, the first file contains all the valid events collected, and subsequent files are made for each data frame. Each data frame is queued separately reconstruction, and the entire framed data set is submitted at the same time for reconstruction as a batch job. Just prior to reconstruction, a random access image file is created with all the known header information filled in. As the images are reconstructed, they are written into this file. The frame headers are also updated during reconstruction when the peak in the image is multiplied by the slice scale factor, and the decay-since-injection factor, and then divided by the effective image time in order to scale the image to $\mathrm{Bq} / \mathrm{cc}$. The images consist of unsigned bytes which are multiplied by the same slice-specific scale factor to provide a quantitative image.

\section{F) Reconstruction and Display}

The ANIPET reconstruction software allows both 2D and 3D mode scans to be reconstructed using variable energy discrimination and coincident-crystal range settings. These parameters filter out random and scattered events.

\section{1) 2D-Axial Mode}

Data collected during 2D-axial mode scans can be formed into images in real-time. The memory buffer which contains the list-mode data is shared by the control and acquisition programs. This program uses the LUTs to determine the two coincident crystals and then back projects a line-of-response between the precise positions of the two crystals at the time the event was recorded. The exact position is determined by interpolating the translation stage's position with respect to the start of the block in which that event is recorded. The image display format for longitudinal studies is 512 by 128 pixels of $0.5 \times 0.5 \mathrm{~mm}$. The image display program produces seven planar images of the average activity distribution in seven equi-volume horizontal planes between the detectors. The image display program has two of these image buffers displayed simultaneously allowing the user to observe temporal tracer distribution changes or to compare two different studies.

\section{2) 3D-Rotary Mode}

Reconstruction of scans done in the 3D-rotary mode use the reconstruction technique developed by Andrew Reader [9] [10], who provided the source code for our project. At present this reconstruction program has been fully integrated into the
ANIPET software and allows for the choice of FAIR (fast iterative reconstruction), FORE+FBP (Fourier rebinning + 2D filtered backprojection), FORE+OSEM (ordered subsets expectation maximization) and BPF (backprojection then filter) reconstruction algorithms. A set of 64 image slices can be reconstructed in 3 minutes with the FORE+FBP algorithm. The BPF technique allows the direct reconstruction of list mode data by inverting the normal order of the filtering and back projecting steps. This technique allows the data to be back projected and then filtered. The advantage of this is that there is no reason to format list mode data into sinogram is prior to reconstruction. The very large number of detector elements and positions makes the sinogram matrix very large.

\section{3) Image Display Functions}

The basic image display program for $2 \mathrm{D}$ or $3 \mathrm{D}$ images allows the user to view all slices ( 7 or 64 respectively) from any frame, zoom in on any frame and display either trans-axial or axial profiles. A region-of-interest tool allows the user to identify up to four circular regions on any slice and generate time activity curves (TACs) through all the frames of a dynamic scan. The TAC files can be exported to other programs where they are combined with blood sample data in order to calculate regional rate constants.

\section{EXPERIMENTAL METHODS}

\section{A) System Timing Resolution}

The system coincidence timing resolution was measured by setting up the detectors to detect coincidence events from a $0.25 \mathrm{MBq}{ }^{68} \mathrm{Ge}$ point source at a distance of $80 \mathrm{~mm}$ in between each detectors. Each detected event evokes a timing signal from the last PMT-dynode of each detector that is sent to a constant

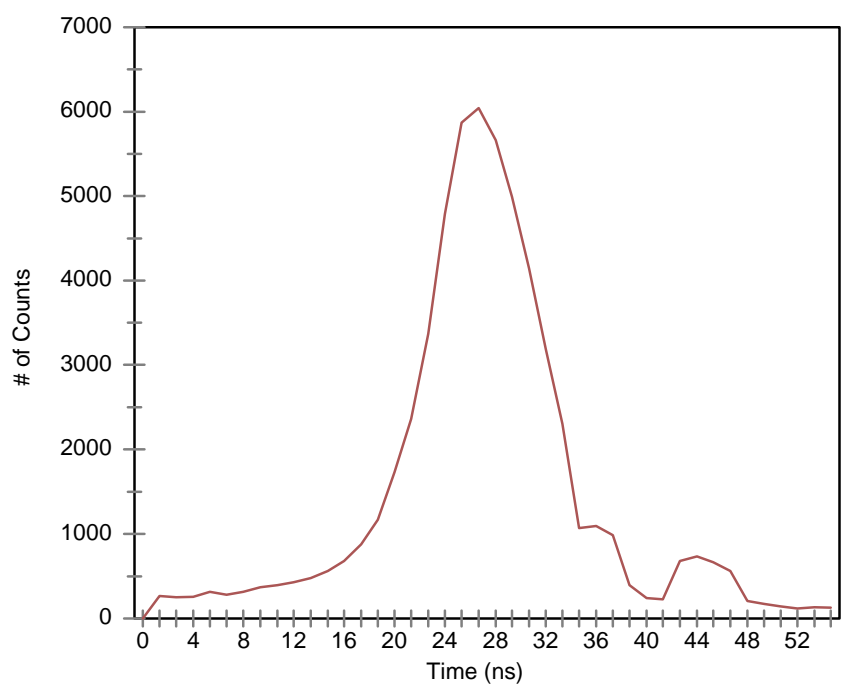

Figure 2: Coincidence Timing Spectra 


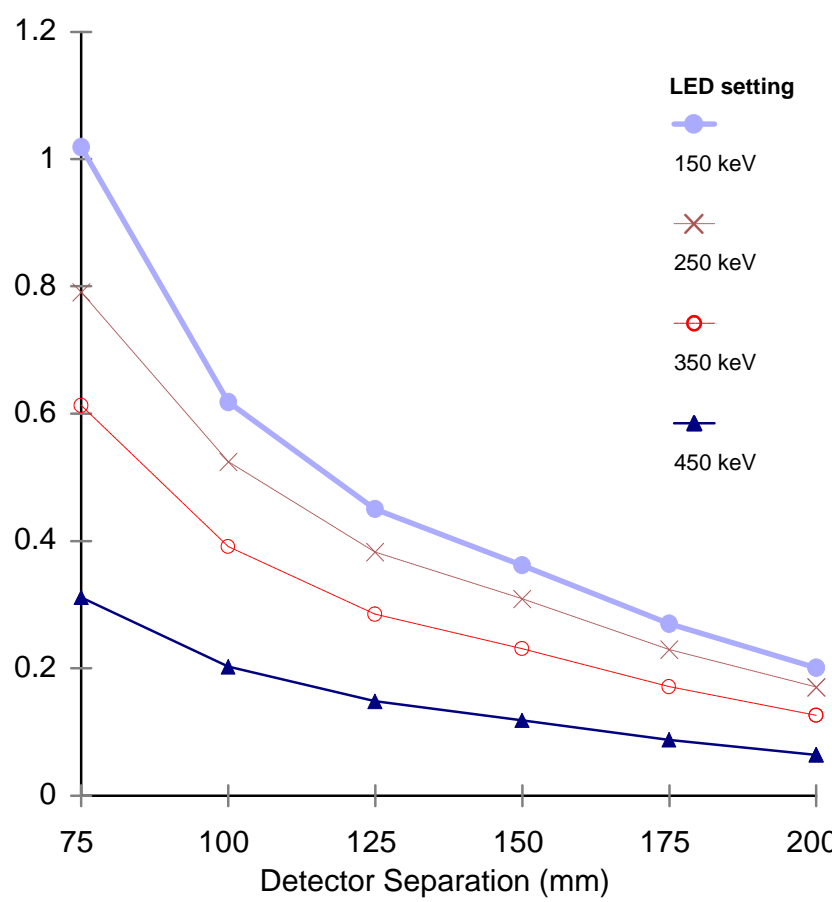

Figure 3: System efficiency as a function of detector separation; plotted for four different low energy discriminator (LED) settings.

fraction discriminator, which in turn generates timing pulses. The timing pulses were processed by a fast-slow triggering of a calibrated time-to-amplitude converter (TAC). The coincidence timing spectrum was read out using a multi-channel analyzer. The count rate and the energy threshold were set to minimize the effect of random events.

\section{B) System Efficiency}

The system efficiency was measured by placing a 0.25 $\mathrm{MBq}{ }^{68} \mathrm{Ge}$ point source at the center of the FOV and recording the number of counts detected in a 200 second period. This was
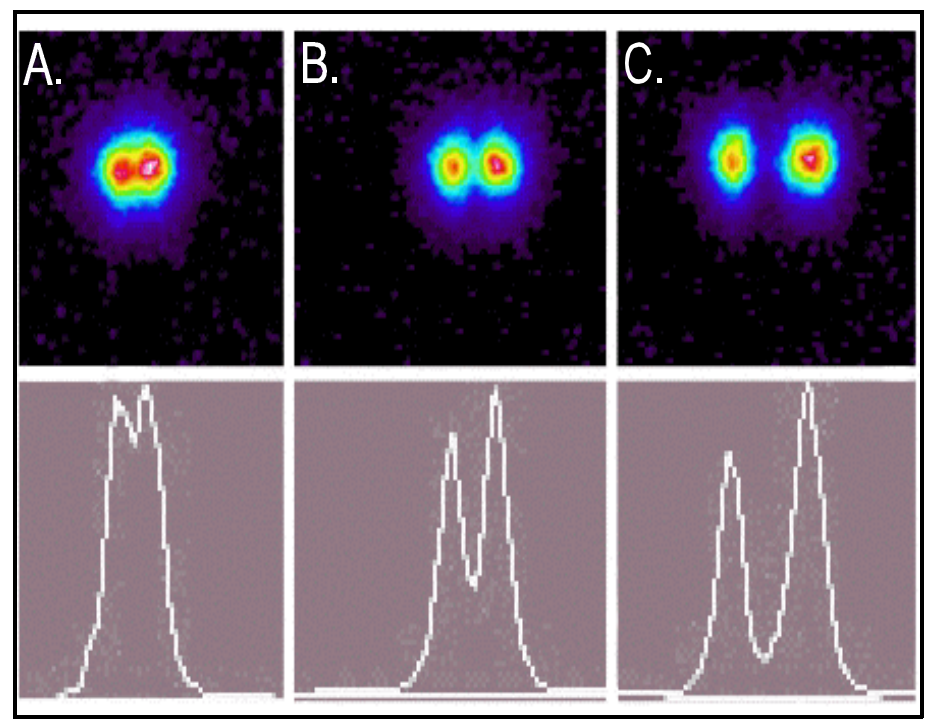

Figure 4: Reconstructed images of 2D-mode scans of two point sources with a separation of A) $4 \mathrm{~mm}$, B) $6 \mathrm{~mm}$, C) $10 \mathrm{~mm}$. Activity profiles through the center of each source are displayed below. measured for different detector separations from 75 to $200 \mathrm{~mm}$ Table 1: Summary of ANIPET Specifications

\begin{tabular}{|c|c|c|}
\hline Mode & 2D & 3D \\
\hline Detector Separation (mm) & $75-200$ & $75-200$ \\
\hline Efficiency (LED=250 keV) & $0.8-0.1 \%$ & $0.8-0.1 \%$ \\
\hline Timing Resolution (ns) & 10 & 10 \\
\hline Axial FOV (mm) & 256 & 54 \\
\hline Trans-Axial FOV (mm) & 54 & $59-84$ \\
\hline Spatial FWHM Resolution (mm) & 2.8 & 3.2 \\
\hline
\end{tabular}

and for different low energy discriminator settings from 150 to $450 \mathrm{keV}$. The high energy discriminator was kept constant at 750 $\mathrm{keV}$ because we are only interested in the signal-to-low energy noise ratio. The fraction of emitted annihilation photons detected by the scanner was plotted as a function of detector separation and low energy discrimination.

\section{C) System Spatial Resolution}

Evaluation of the system spatial resolution in the both 2D and 3D scanning modes was based on the FWHM of an activity profile through an image of a $0.75 \mathrm{~mm}$ diameter ${ }^{68} \mathrm{Ge}$ point source. Our ${ }^{68} \mathrm{Ge}$ sources are contained in a nickel casing that has a diameter of $3 \mathrm{~mm}$.

Figures 4 and 5 were acquired by placing two point sources inside a plastic Resolution Tool, which has holes drilled precisely at 4,6,and $10 \mathrm{~mm}$ separations, to accurately monitor the spatial scale factor of the reconstructed images. For 2D and 3D mode scans the tool was placed on the center axis of the detectors, which were separated by $200 \mathrm{~mm}$, and scanned for 200 seconds. For the 2D scan the bed movement was set to scan

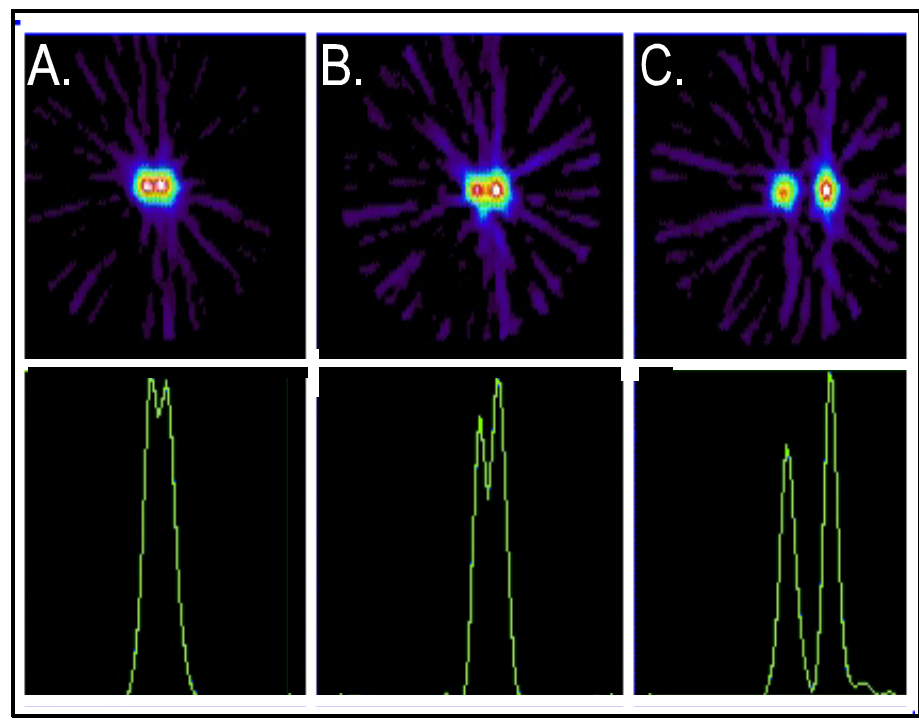

Figure 5: Reconstructed images of 3D-mode scans of two point sources with a separation of A) $4 \mathrm{~mm}$, B) $6 \mathrm{~mm}$, C) $10 \mathrm{~mm}$. Activity profiles through the center of each point source are depicted below. 
an $80 \mathrm{~mm}$ axial FOV at the speed of $4 \mathrm{~mm} /$ second. Scans were performed for source separations of 4,6 , and $10 \mathrm{~mm}$, and reconstructed with a LED of $350 \mathrm{keV}$.

Before the resolution experiments were started, the accuracy of the reconstruction scaling factors was verified for both horizontal and vertical spatial distance. This was accomplished by doing a scan with the sources $10 \mathrm{~mm}$ apart and observing that the distance between the source centers in the reconstructed image was in fact $10 \mathrm{~mm}$.

\section{PRELIMINARY RESULTS}

\section{A) System Timing Resolution}

The timing spectrum is shown in Fig. 1 exhibits a FWHM of 10 ns. More than $75 \%$ of the counts fell within a window width of $10 \mathrm{~ns}$. This is very acceptable for typical coincidence window setting of $13 \mathrm{~ns}$.

\section{B) System Efficiency}

Figure 3 is a plot of the percentage of emitted annihilation photons detected by the system as a function of the detector separation and low energy discrimination. As the detector separation was increased from 75 to $200 \mathrm{~mm}$ the efficiency decreases from $1.0 \%$ to $0.2 \%$ when the low energy window is set to $150 \mathrm{keV}$. The system efficiency in the 2D and 3D modes are equivalent and vary only as a function of detector separation and energy discrimination.

\section{C) System Spatial Resolution}

2D mode scans of two point sources separated by $10 \mathrm{~mm}$ produced an images that was easily discernable. At $4 \mathrm{~mm}$ the image of 2 point sources was not as well defined (Fig. 4). In the 2D mode the FWHM of a profile through a point source in the centre of the FOV was $2.8 \mathrm{~mm}$, and does not vary as a function of translation speed or position in the detector FOV.

The spatial resolution of the 3D-scans of points sources in the centre of the FOV varies from $3.2-4.5 \mathrm{~mm}$ as the coincident-crystal range was increased from 5 to 72 . At a constant coincident-crystal range of 20 the spatial resolution did not vary as a function of axial position, however trans-axial displacement off-centre caused a degradation from 3.5 to $4.3 \mathrm{~mm}$ as a point source was displaced from 0 to $25 \mathrm{~mm}$ off-centre.

\section{DISCUSSION}

The ANIPET system has been designed to provide the versatility required for scanning tracer distribution in a wide range of laboratory animals. This evaluation presents some of the most important performance measurements. Further work is needed to map out the spatial resolution throughout the field of view. We have however dealt with problems relating to the electrical interference between the stepping motors and detector timing signals, and improved the detectors' stability and field of view during the last year for which the system has been in operation.

\section{Acknowledgments}

This project was supported by MRC grant MTI13614 and MST37557.

\section{REFERENCES}

[1] Hichwa R. "Are Animal Scanners Really Necessary for PET?," J Nucl. Med. 35, pp 1396-7, 1994.

[2] Muck-Seler D, Jevric-Causevic A, Diksic M. "Influence of Fluoxitine on Regional Serotonin Synthesis in the Rat Brain," J Neurochem. 67, pp.2434-2442, 1996.

[3] Thompson CJ, Sciascia P, Murthy K, Kecani S, Nikkinen K, del Campo E, Corbett J-F, Bercier Y, Diksic M, Cumming P. "“ANIPET' a Versatile PET Scanner for Imaging Small Animals," Paper \# M6-33 Proceedings of the 1998 IEEE Medical Imaging Conference, Toronto, 1998.

[4] Weber S, Herzog H, Coenen HH, Cremer M, Engels R, Hamacher K, Kehren F., et al. "Evaluation of TierPET System," IEEE Nuclear Science Symposium \& Medical Imaging Conference Reeord,1999.

[5] Cherry S R, Chatzioannou A, Shao Y, Silverman R W, Meadors and Phelps M E, "Brain Imaging in Small Animals Using MieroPEY," In: Quantitative Functional Imaging with Positron Emission Tomography, Academic Press, pp 3-9, 1998.

[6] Lecomte, R. Cadorette, J. Richard, P. et al. "Design and Engineering Aspects of a High Resolution Positron Tomograph for Small Animal Imaging," IEEE Trans Nucl Sci. NS-41 \#4: pp. 1446-1452, 1994.

[7] Robar J L, Thompson C J, Murthy K, Clancy R, Bergman AM. "Construction and Calibration of Detectors for High Resolution Metabolic Breast Cancer Imaging," Nucl. Inst. and Meth. in Phys. Res. A 392, pp. 402-6,1997.

[8] Murthy K, Aznar M, Bergman AM, Thompson CJ, et al.,. "Positron Emission Mammographic Instrument: Initial Results," Radiology, 215, pp. 280-5, 2000.

[9] Townsend DW, Byars LG, Defrise M, Geissbuhler A et al. "Design and performance of a rotating positron tomograph, RPT-2 ${ }^{\circ}$," IEEE Nuclear Science Symposium \& Medical Imaging Conference Record, pp. 1058-1062, 1994.

[10] Zhang N, Thompson CJ, Nguyen KQ, Murthy K. "Improving the Performance of Small Planar Detectors for Dedicated PET Instruments," IEEE Nuclear Science Symposium \& Medical Imaging Conference Record, \#IEEE461, 2000.

[9] Reader A J, Erandsson K, Flower M A, and Ott R J. "Fast iterative reconstruction for low statistics positron volume imaging," Proceedings of the 1997 International Meeting on Full 3D Image Reconstruction, Pittsburgh, pp. 166-69, 1997.

[10] Reader A, Visvikis D, Flower M A and Ott R J. "Intercomparison of four reconstruction techniques for position volume imaging with rotating planar detectors," Proceedings of the 1997 International Meeting on Full 3D Image Reconstruction, Pittsburgh, pp. 201-4, 1997 